\title{
Thermomechanical transient analysis of a thick-hollow FGM cylinder
}

\author{
Behrouz Takabi*
}

Texas A\&M University, Department of Mechanical Engineering, 3123 TAMU, College Station, TX 77843, United States

\begin{tabular}{l}
\hline A R T I C L E I N F O \\
\hline Article history: \\
Received 6 April, 2015 \\
Accepted 10 October 2015 \\
Available online \\
11 October 2015 \\
\hline Keywords: \\
FGM \\
Hollow cylinder \\
Numerical solution \\
Transient \\
Thermomechanical \\
Tempreture \\
\end{tabular}

\section{A B S T R A C T}

\begin{abstract}
This paper presents an analytical and a numerical thermomechanical investigation of a thickwalled cylinder made of the functionally graded materials (FGMs). The hollow cylinder is subjected to a pressure and a thermal load and the properties of this material are varying across the thickness from the inner face that is a ceramic to the outer one which is a metal. After validating the current results by the analytical results, the thermomechanical behavior of the cylinder in the transient condition is investigated and the hoop and radial stresses and also temperature distribution are reported. The results reveal that the use of FG material causes a decrease in the stress; thus, it significantly improves thermomechanical behavior of the domain, thanks to using FG materials.
\end{abstract}

\section{Nomenclature}

\begin{tabular}{|lllllll|}
\hline$r$ & Radios & $\rho$ & Density & $P_{b}$ & External pressure & $l$ \\
$T$ & Temperature & $\sigma_{r r}$ & Radial stress & $T_{a}$ & Inner temperature & \\
$P$ & Pressure & $\sigma_{\theta \theta}$ & Hoop stress & $T_{b}$ & Outer temperature \\
$E$ & Young modulus & $C$ & Specific heat capacity & $a$ & Inner radius \\
$u$ & Displacement & $K$ & Thermal conductivity & $b$ & Outer radius \\
$\varepsilon$ & Strain & $\alpha$ & Thermal expansion & $f$ & Volume fraction of FGM \\
$v$ & Poisson ratio & $P_{a}$ & Internal pressure & $n$ & Power factor of FGM & \\
\hline
\end{tabular}

\section{Introduction}

Nowadays, functionally graded materials (FGMs) are playing an important role in the design of complicated and modern structures. FGMs have a range of specifications which caused scientists tend to study on these materials. The most important exclusivity of FGMs is the ability of change in properties

* Corresponding author. Tel.: +19792188169

E-mail addresses: btakabi@tamu.edu (B. Takabi) 
from one surface to the other, gradually and continuously. Therefore, these advanced materials can be utilized in structures which need to have varying properties in certain directions. One of the common applications of this material is in a cylinder that should resist high thermal stresses in the inner surface, while the outer face is under high mechanical loads. Therefore, FGM cylindrical structures have been widely employed in automobile and nuclear and aerospace industries.

In order to design and analysis of structures made of FGM, various simulation methods including numerical and analytical approaches have been proposed so far (Aihara et al., 1998; Salavati et al., 2014; Vimal et al., 2014). Most of the studies have focused on assessing thermal stresses and also the deformation of the domain, such as the use of FGMs for controlling of thermal deformations (Wetherhold et al., 1996), the investigation of thermal stresses and deformation in shells made of FGM under thermal loads (Takezono et al., 1996; Zhang et al., 1994).

Kandil et al. (1995) performed a computational study on a thick-walled cylinder subjected to dynamic internal temperature gradient. They reported thermal stresses and also the temperature distribution within the cylinder wall. Ghosn and Sabbaghian (1982) achieved the general solutions of governing equations in the Laplace transform domain for a one dimensional axisymmetric thermoelastic material. Moreover, a transversely isotropic hollow cylinder of finite length has solved using a direct power series approximation by Chen (1983). Shahani and Nabavi (2007) analytically investigated the thermoelastic behavior of a thick-walled cylinder. For this purpose, they used the finite Hankel transform. Besides, they applied the time dependent thermal boundary conditions on the inner surface of the cylinder. Moreover, for the mechanical boundary conditions, they considered that traction is prescribed on the inner surface and the fixed displacement boundary condition on the outer one; further, they examined the case that tractions are prescribed on both the inner and outer surfaces of the hollow cylinder. Therefore, the transient thermal response of the cylinder was derived and then the quasi-static structural problem was solved and closed form relations were extracted for the thermal stresses.

Kardomateas $(1989,1990)$ performed an analytical study on an orthotropic hollow cylinder subjected to general form of constant thermal boundary conditions, using Hankel asymptotic expansions for Bessel functions. Yee and Moon (2002) performed a study on the transient, plane quasi-static thermal stress analysis of an orthotropic hollow cylinder subjected to an arbitrary initial temperature distribution and homogeneous thermal boundary conditions. The thermoelastic solution was obtained by a stress function approach. Jabbari et al. (2007) suggested a general theoretical analysis of three-dimensional mechanical and thermal stresses for a short hollow FGM cylinder by the generalized Bessel function and Fourier series.

Goshima and Miyao (1991) analytically solved a long hollow circular cylinder subjected to transient internal heat generation due to x-ray radiation, while there is convection on its inner and outer surfaces. For this purpose, they used Laplace transform and Green's function. Bayat et al. (2012) performed a thermo-mechanical analysis on a hollow FGM sphere. They investigated the effect of non-homogeneity in FGM thick sphere by choosing a dimensionless parameter. They also reported that by changing the value of that parameter, the properties of FGM can be so modified that the lowest stress levels are reached.

Most of the literatures have performed the analytical solution of some simple geometries made of FGM, considering simplified assumptions, such as employing Hankel transform (Goshima \& Miyao, 1991) or Laplace transform (Sutradhar et al., 2001). In this work, a thick-hollow FGM cylinder subjected to both internal and external pressures and also under thermal loads is solved. Moreover, in this study, the properties of material are varying from the inner layer that is a ceramic to the outer face which is a metal continuously and gradually in the thickness direction. The variable properties are density, specific heat capacity and thermal conductivity. Then, the transient thermal distribution and also stress distribution including the hoop, radial stresses are calculated. Then the current results are validated by an analytical solution in (Shahani \& Nabavi, 2007). 


\section{Analysis and formulation}

For the analysis, a hollow cylinder made of functionally graded material with inner and outer radius $a$ and $b$, respectively is considered. This cylinder is long enough in the axial direction for satisfying the plane strain condition. The properties of this material such as $\rho, c$ and $k$ are varying from the inner to outer face. However, because the impact of $v$ (Poisson ratio) is negligible on the stresses, $v$ is assumed to be constant throughout the thickness. This assumption has used in numerous studies such as Erdogan (1995), Horgan and Chan (1999), Chen and Erdogan (1996). Then the temperature distribution is found and radial and hoop stress also are calculated and discussed.

For the computational approach, the assumption of multi-layer is used for FGM in the thickness direction. In other words, the geometry consists of some layers and each layer is formed by some elements. The properties in each layer are assumed to be constant which is a common and usual approach in this field.

\subsection{Temperature distribution}

The Temperature in any element is calculated by linear form of shape function:

$$
\begin{aligned}
& T=[N]\{T\}^{e} \\
& {[N]=\left[\begin{array}{ll}
\frac{r_{i+1}-r}{l} & \frac{r-r_{i}}{l}
\end{array}\right]}
\end{aligned}
$$

and

$$
l=r_{i+1}-r_{i}
$$

and also we have

$$
\frac{\partial T}{\partial t}=[N]\{\dot{T}\}^{e}
$$

According to Fourier equation (Eq. (3)) and considering the plane strain condition (the cylinder is long enough), the axisymmetric equation will be as Eq. 4:

$$
\begin{aligned}
& \frac{1}{r} \frac{\partial}{\partial r}\left(K r \frac{\partial T}{\partial r}\right)+\frac{1}{r^{2}} \frac{\partial}{\partial \varphi}\left(K \frac{\partial T}{\partial \varphi}\right)+\frac{\partial}{\partial z}\left(K \frac{\partial T}{\partial z}\right)+\dot{q}=\rho C_{p} \frac{\partial T}{\partial t}, \\
& \frac{1}{r} \frac{\partial}{\partial r}\left(K r \frac{\partial T}{\partial r}\right)=\rho C_{p} \frac{\partial T}{\partial t},
\end{aligned}
$$

where $\rho$ is density, $C$ specific heat capacity and $K$ thermal conductivity.

Using Galerkin method for Eq. (4), we have:

$$
\int[N]^{T}\left\{\frac{1}{r} \frac{\partial}{\partial r}\left(K r \frac{\partial T}{\partial r}\right)-\rho c \frac{\partial T}{\partial t}\right\} 2 \pi r d r=0
$$

Simplifying Eq. (5) yields

$$
\begin{aligned}
& {\left[K \int\left\{-[N]^{T} \frac{\partial[N]}{\partial r}+[N]^{T} \frac{\partial[N]}{\partial r}+\frac{\partial[N]^{T}}{\partial r} r \frac{\partial[N]}{\partial r}\right\} d r\right]\{T\}^{e}} \\
& +\rho c \int\left[[N]^{T} r[N] d r\right]\{\dot{T}\}^{e}=\left.K[N]^{T} r \frac{\partial T}{\partial r}\right|_{r_{i}} ^{r_{i+1}}
\end{aligned}
$$


Employing finite element in matrix form gives us Eq. (7).

$$
[K]\{T\}^{e}+[C]\{\dot{T}\}^{e}=\{f\}
$$

where

$$
\begin{aligned}
{[K] } & =K \int_{r_{i}}^{r_{i+1}} \frac{\partial[N]^{T}}{\partial r} r \frac{\partial[N]}{\partial r} d r=\frac{K}{2 l}\left[\begin{array}{cc}
r_{i}+r_{i+1} & -r_{i}-r_{i+1} \\
-r_{i}-r_{i+1} & r_{i}+r_{i+1}
\end{array}\right], \\
{[C] } & =\rho c \int_{r_{i}}^{r_{i+1}}[N]^{T} r[N] d r=\rho c \int_{r_{i}}^{r_{i+1}}\left[\begin{array}{c}
\frac{r_{i+1}-r}{l} \\
\frac{r-r_{i}}{l}
\end{array}\right] r\left[\frac{r_{i+1}-r}{l} \frac{r-r_{i}}{l}\right] d r \\
& =\frac{\rho c l}{12}\left[\begin{array}{cc}
3 r_{i}+r_{i+1} & r_{i}+r_{i+1} \\
r_{i}+r_{i+1} & r_{i}+3 r_{i+1}
\end{array}\right], \\
\{f\} & =\left.K\left[\begin{array}{c}
\frac{r_{i+1}-r}{l} \\
\frac{r-r_{i}}{l}
\end{array}\right] r \frac{\partial T}{\partial r}\right|_{r_{i}} ^{r_{i+1}}=K\left[\begin{array}{c}
-\left.r_{i} \frac{\partial T}{\partial r}\right|_{r_{i}} \\
\left.r_{i+1} \frac{\partial T}{\partial r}\right|_{r_{i+1}}
\end{array}\right] .
\end{aligned}
$$

Moreover, Eq. 11 shows the relation between the place and the time for temperature $(\mathrm{T})$

$$
[K]\left\{\begin{array}{c}
T_{i}^{p} \\
T_{i+1}^{p}
\end{array}\right\}+[C]\left\{\begin{array}{c}
\dot{T}_{i}^{p} \\
\dot{T}_{i+1}^{p}
\end{array}\right\}=\{f\},
$$

where $p$ is a time parameter and $i$ stands for a place parameter.

In Eq. (11), backward method is used for time derivative of temperature. It should be noted that these Eqs. are for one element; and these matrixes must be assembled into a general matrix.

$$
T_{j}=T_{j+1}, \quad K_{j} \frac{\partial T}{\partial r}=K_{j+1} \frac{\partial T}{\partial r}
$$

Using continuity condition in layer boundaries (Eq. (12)), vector $\{f\}$ is eventually assembled as:

$$
\{F\}=\left\{\begin{array}{c}
-\left.K_{1} r_{1} \frac{\partial T}{\partial r}\right|_{r=r_{1}} \\
\vdots \\
\vdots \\
\circ \\
\left.K_{N} r_{N} \frac{\partial T}{\partial r}\right|_{r=r_{N}}
\end{array}\right\}
$$




\subsection{Stress distribution}

Using the plane strain assumption and axisymmetry, the strain displacement follows the below equations:

$$
\begin{aligned}
& \varepsilon_{r r}=\frac{\partial u}{\partial r}, \quad \varepsilon_{\theta \theta}=\frac{u}{r}, \quad \varepsilon_{z z}=0 \\
& \sigma_{r r}=(2 \mu+\lambda) \varepsilon_{r r}+\lambda\left(\varepsilon_{\theta \theta}+\varepsilon_{z z}\right)-\beta\left(T-T_{0}\right) \\
& \sigma_{\theta \theta}=(2 \mu+\lambda) \varepsilon_{\theta \theta}+\lambda\left(\varepsilon_{r r}+\varepsilon_{z z}\right)-\beta\left(T-T_{0}\right) \\
& \sigma_{r r}=(2 \mu+\lambda) \frac{\partial u}{\partial r}+\lambda\left(\frac{u}{r}\right)-\beta\left(T-T_{0}\right) \\
& \sigma_{\theta \theta}=(2 \mu+\lambda) \frac{u}{r}+\lambda\left(\frac{\partial u}{\partial r}\right)-\beta\left(T-T_{0}\right)
\end{aligned}
$$

In the radial direction, the governing equation is:

$$
\frac{\partial^{2} \sigma_{r r}}{\partial r^{2}}+\frac{1}{r} \frac{\partial \sigma_{r \theta}}{\partial \theta}+\frac{\partial \sigma_{z r}}{\partial z}+\frac{1}{r}\left(\sigma_{r r}-\sigma_{\theta \theta}\right)=\rho \ddot{u}
$$

and after simplifying the governing equation according to the assumptions used in this problem, we have

$$
\frac{\partial^{2} \sigma_{r r}}{\partial r^{2}}+\frac{1}{r}\left(\sigma_{r r}-\sigma_{\theta \theta}\right)=\rho \ddot{u}
$$

Eventually, the equation in terms of displacement is

$$
(2 \mu+\lambda)\left(\frac{\partial^{2} u}{\partial r^{2}}+\frac{1}{r} \frac{\partial u}{\partial r}-\frac{u}{r^{2}}\right)=\beta \frac{\partial T}{\partial r}+\rho \ddot{u}
$$

where

$$
\begin{aligned}
& \mu=G=\frac{E}{2(1+v)}, \\
& \lambda=\frac{E v}{(1+v)(1-2 v)}, \\
& \beta=(3 \lambda+2 \mu) \alpha,
\end{aligned}
$$

where $E$ stands for Young modulus, $v$ is Poisson ratio and $\alpha$ is thermal expansion.

Employing the similar approach for temperature distribution (Galerkin method) and assembling the related matrix and applying continuity condition in layer boundaries (Eq. (21)), then Eq. (19) is solved and stress distribution is found.

$$
u_{j}=u_{j+1}, \quad\left(\sigma_{r r}\right)_{j}=\left(\sigma_{r r}\right)_{j+1}
$$

\section{Simulation of properties varying}

In this paper, it is considered that the material properties are varying from inner face (ceramic layer) gradually to the outer one (metal layer) in the thickness direction according to the volume fraction law. 
For this purpose, the volume fraction law, as shown in Eq. (22), which is more accurate and comprehensive than power law is employed.

$$
\lambda=\lambda_{c}(1-f)+\lambda_{m} . f
$$

where $\lambda$ is any property of FGM varying and $\lambda_{c}$ is the property of the ceramic and $\lambda_{m}$ stands for the metal. In addition, $f$ can be defined as:

$$
f=\left(\frac{r-a}{b-a}\right)^{n}
$$

As defined earlier $a$ and $b$ are the inner and outer radius, respectively and $r$ is the location that its property is $\lambda$. Varying $n$ can be used in different profiles of properties verification. For example, very small values of $n$ demonstrate that the property is constant and equal to that of the metal. In this work, $\rho, K$ and $C$ are varying based on the above law, while $v$ is constant. Therefore,

$$
\begin{aligned}
& \rho(r)=\rho_{c}(1-f)+\rho_{m} \cdot f, \\
& K(r)=K_{c}(1-f)+K_{m} . f, \\
& C(r)=C_{c}(1-f)+C_{m} \cdot f .
\end{aligned}
$$

\section{Results and discussion}

To ensure the validity of the current results, they are compared to the analytical solution by Shahani and Nabavi (2007). Hence, the material properties of functionally graded materials and the geometry are the same and their value are presented in Table 1.

Table 1. Properties of material in the inner and the outer surface

\begin{tabular}{lllllll}
\hline & $\rho\left(\frac{\mathrm{kg}}{\mathrm{m}^{3}}\right)$ & $\begin{array}{c}E \\
(\mathrm{GPa})\end{array}$ & $K\left(\frac{\mathrm{W}}{\mathrm{mK}}\right)$ & $\begin{array}{l}\alpha \times 10^{6} \\
(1 / K)\end{array}$ & $C\left(\frac{\mathrm{kJ}}{\mathrm{kgK}}\right)$ & $\boldsymbol{V}$ \\
\hline$a=1 m$ & 5730 & 210 & 3.03 & 10.2 & 450 & 0.3 \\
$b=2 m$ & 7800 & 208 & 2 & 12 & 25.6 & 0.3 \\
\hline
\end{tabular}

The transient temperature distribution and the hoop and radial stress, respectively are verified by Shahani and Nabavi (2007) in Figs. (1-3. For all these figures, the time is the same (1000 sec).

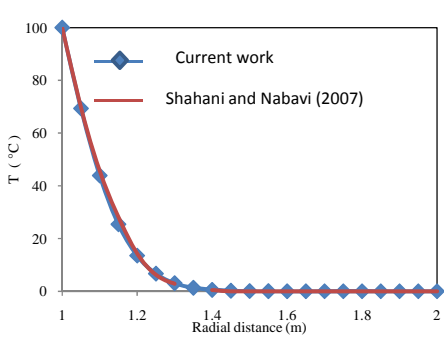

Fig.1. Temperature distribution for $t=1000 \mathrm{sec}$

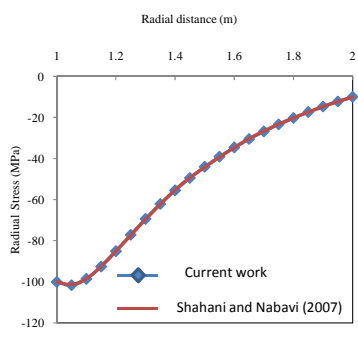

Fig. 2. Radial stress distribution for $t=1000 \mathrm{sec}$

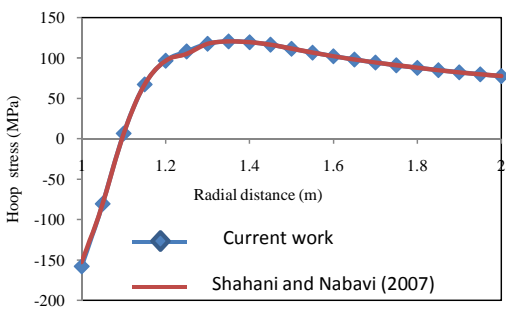

Fig. 3. Hoop stress distribution for $t=1000 \mathrm{sec}$

In Figs. 4-7, the effect of $n$ on the temperature distribution has been studied. In this case, the metal is $\mathrm{Al}$ and the ceramic is $\mathrm{SiO}_{2}$ and their properties are defined in Table 2. Moreover, the boundary conditions are: 


$$
\begin{aligned}
& T_{1}=100{ }^{\circ} \mathrm{C}, \mathrm{T}_{2}=0{ }^{\circ} \mathrm{C} \\
& P_{1}=100 \mathrm{MPa}, P_{2}=10 \mathrm{MPa}
\end{aligned}
$$

and 1 is the inner face and 2 stands for the outer face.

Table 2. Properties of material

\begin{tabular}{lllllll}
\hline & $\rho\left(\frac{k g}{m^{3}}\right)$ & $E(G P a)$ & $K\left(\frac{W}{m K}\right)$ & $\alpha \times 10^{6}(1 / K)$ & $C\left(\frac{J}{k g K}\right)$ & $v$ \\
\hline $\mathrm{r} 1=0.1 \mathrm{~mm}\left(\mathrm{SiO}_{2}\right)$ & 2600 & 150 & 1.38 & 3 & 745 & 0.3 \\
$\mathrm{r} 1=0.2 \mathrm{~mm}(\mathrm{Al})$ & 2702 & 70 & 237 & 23 & 903 & 0.3 \\
\hline
\end{tabular}

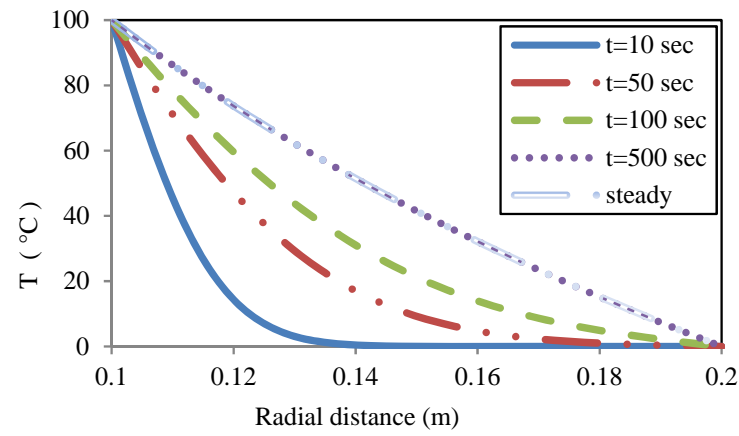

Fig. 4.Temperature distribution for $n=0.00001$

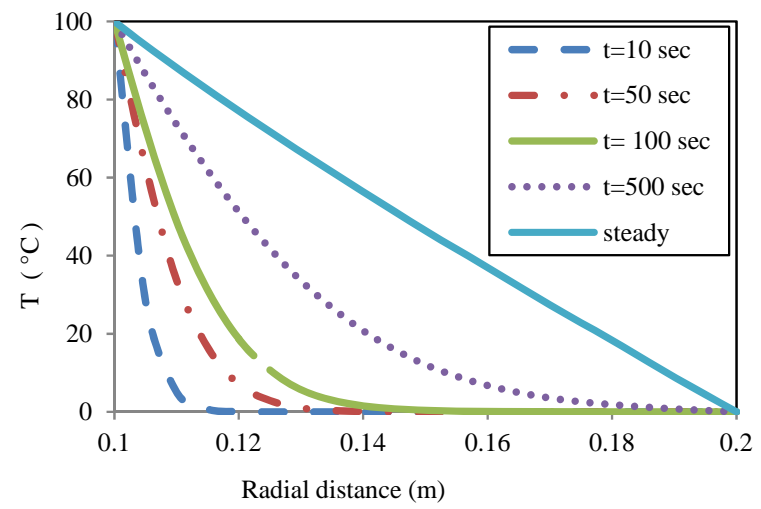

Fig. 6. Temperature distribution for $n=1$

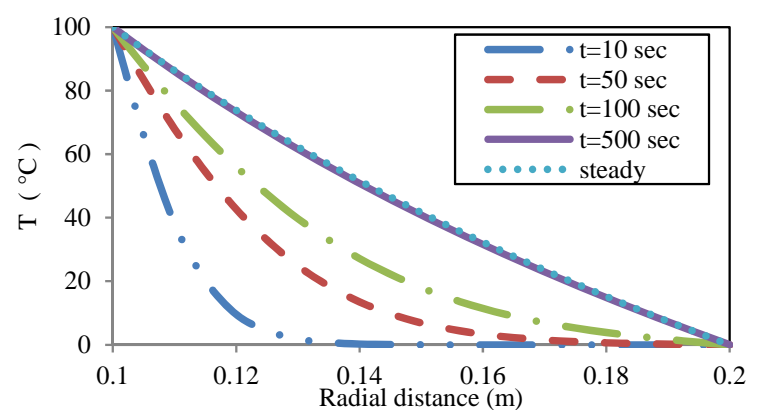

Fig. 5. Temperature distribution for $n=0.01$

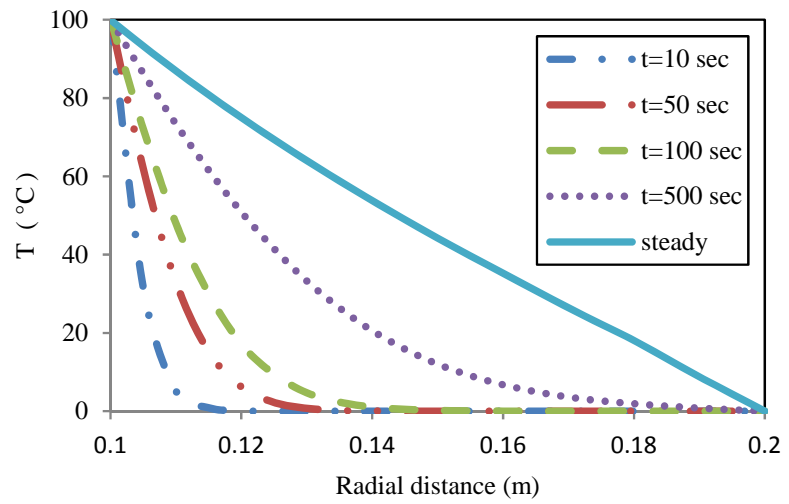

Fig. 7. Temperature distribution for $n=5$

According to Figs. (4-7), it can be found out that by passing time, the temperature distribution goes to a linear form and after a large time (steady state), it becomes a straight line. This behavior is reasonable, because in any isotropic material, the steady state temperature distribution between the inner and the outer surface must be linear. Further, these figures show that the average of the temperature in FG material is less than that of a metal one; and this leads to this fact that the thermal stress in the FGM is less than that in the metal. Also, in Fig. 4 which is based on a very small $n(n=0.00001)$ that means the thermomechanical behavior is near to that in the metal, temperature profiles tend to become a linear form; hence, the average temperature in this case is more than that in Fig. 7 with larger $n$.

\section{Conclusion}

In this work, the thermomechanical analysis of a thick hollow cylinder made of functionally graded materials was performed. The thick hollow cylinder is under pressure and thermal load and the 
properties are not constant. The properties are varying according to the power law somehow the inner surface of the cylinder is made of a ceramic which is $\mathrm{SiO}_{2}$ and the outer face is from a metal that is $\mathrm{Al}$. Then the results obtained from the present study were validated with the analytical solutions by Shahani and Nabavi (2007). After that, the thermomechanical stresses were assessed under the transient condition. Furthermore, the effect of $n$ (defined in power law) on thermomechanical behavior of the FGM cylinder was investigated. The results demonstrate that the use of FG material that is a typical composition of a metal and a ceramic leads to a noticeable improvement of thermomechanical behavior.

\section{References}

Aihara, T. (1998). Molecular dynamics simulation on elastic behavior of Ni/Ni 3 Al interface with graded structure. Japan Institute of Metals, Journal, 62(11), 978-985.

Bayat, Y., Ghannad, M., \& Torabi, H. (2012). Analytical and numerical analysis for the FGM thick sphere under combined pressure and temperature loading. Archive of Applied Mechanics, 82(2), 229-242.

Chen, Y. F., \& Erdogan, F. (1996). The interface crack problem for a nonhomogeneous coating bonded to a homogeneous substrate. Journal of the Mechanics and Physics of Solids, 44(5), 771-787.

Chen, P. Y. P. (1983). Axisymmetric thermal stresses in an anisotropic finite hollow cylinder. Journal of Thermal Stresses, 6(2-4), 197-205.

Erdogan, F. (1995). Fracture Mechanics of Functionally Graded Materials. MRS Bulletin, 20(01), 43-44.

Ghosn, A. H., \& Sabbaghian, M. (1982). Quasi-static coupled problems of thermoelasticity for cylindrical regions. Journal of Thermal Stresses, 5(3-4), 299-313.

Goshima, T.\& Miyao K. (1991). Transient thermal stress in a hollow cylinder subjected to x-ray heating and convective heat losses, Nuclear Engineering Design. 125, 267-273.

Horgan, C. O., \& Chan, A. M. (1999). The pressurized hollow cylinder or disk problem for functionally graded isotropic linearly elastic materials. Journal of Elasticity, 55(1), 43-59.

Jabbari, M., Mohazzab, A. H., Bahtui, A., \& Eslami, M. R. (2007). Analytical solution for three-dimensional stresses in a short length FGM hollow cylinder. ZAMM-Journal of Applied Mathematics and Mechanics/Zeitschrift für Angewandte Mathematik und Mechanik, 87(6), 413-429.

Kandil, A., El-Kady, A. A., \& El-Kafrawy, A. (1995). Transient thermal stress analysis of thick-walled cylinders. International journal of mechanical sciences, 37(7), 721-732.

Kardomateas, G. A. (1989). Transient thermal stresses in cylindrically orthotropic composite tubes. Journal of applied mechanics, 56(2), 411-417.

Kardomateas, G. A. (1990). The initial phase of transient thermal stresses due to general boundary thermal loads in orthotropic hollow cylinders. Journal of Applied Mechanics, 57(3), 719-724.

Shahani, A. R., \& Nabavi, S. M. (2007). Analytical solution of the quasi-static thermoelasticity problem in a pressurized thick-walled cylinder subjected to transient thermal loading. Applied Mathematical Modelling, 31(9), 1807-1818.

Salavati, H., Berto, F., \& Alizadeh, Y. (2014). The flow stress assessment of austenitic-martensitic functionally graded steel under hot compression. Engineering Solid Mechanics, 2(2), 83-90.

Sutradhar, A., Paulino, G. H., \& Gray, L. J. (2002). Transient heat conduction in homogeneous and nonhomogeneous materials by the Laplace transform Galerkin boundary element method. Engineering Analysis with Boundary Elements, 26(2), 119-132.

Takezono, S., Tao, K., Inamura, E., \& Inoue, M. (1996). Thermal stress and deformation in functionally graded material shells of revolution under thermal loading due to fluid. JSME international journal. Series A, mechanics and material engineering, 39(4), 573-581.

Vimal, J., Srivastava, R., Bhatt, A., \& Sharma, A. (2014). Free vibration analysis of moderately thick functionally graded skew plates. Engineering Solid Mechanics, 2(3), 229-238.

Wetherhold, R. C., Seelman, S., \& Wang, J. (1996). The use of functionally graded materials to eliminate or control thermal deformation. Composites Science and Technology, 56(9), 1099-1104.

Yee, K. C., \& Moon, T. J. (2002). Plane thermal stress analysis of an orthotropic cylinder subjected to an arbitrary, transient, asymmetric temperature distribution. Journal of applied mechanics, 69(5), 632-640.

Zhang, X. D., Liu, D. Q., \& Ge, C. C. (1994). Thermal stress analysis of axial symmetry functionally gradient materials under steady temperature field. Journal of Functionally Graded Materials, 25,573-581. 